P1.09 DIAGNOSING MYCOPLASMA GENITALIUM: COMPARISON OF FOUR NUCLEIC ACID AMPLIFICATION TESTS AND USE OF THE SPEEDX REAL-TIME PCR ASSAY FOR AZITHROMYCIN RESISTANCE

Charlotte Gaydos, Justin Hardick, Susan Tuddenham, Maria Trent. Johns Hopkins University, Baltimore, USA

10.1136/sextrans-2017-053264.117

Introduction Mycoplasma genitalium (MG) is a sexually transmitted infection (STI) associated with urethritis in men and cervicitis and pelvic inflammatory disease (PID) in women. Azithromycin (AZ) resistance in MG, resulting in treatment failure is an issue. We compared molecular assays for detection of MG in females; a prospective cohort of adolescents with PID and a retrospective group of women at a STD clinic. We tested vaginal samples for AZ resistance with the SpeeDX assay, a real-time PCR, which detects MG and 23S rRNA AZ resistance mutations.

Methods For the prospective study, SpeeDX results were compared to $16 \mathrm{~S}$ rRNA and pdhD real-time PCR assays, and the Aptima MG ASR assay. Samples consisted of dry transported vaginal swab samples $(n=116)$; expressed in water. For the retrospective study we evaluated vaginal samples $(n=289)$ stored in liquid Amies media. Nucleic acid extraction was performed utilising the Roche MagNA Pure LC robot according to manufacturer instructions. The SpeeDX assay was performed according to manufacturer instructions. For the retrospective study, results were compared to the 2 real-time PCR assays for the $16 \mathrm{~S}$ rRNA gene and pdhD gene.

Results For the prospective study, a gold standard of 3 of 4 positive tests defined a true positive. The 16S PCR had 100\% sensitivity (10/10) and $100 \%$ specificity (106/106) Kappa $=1$ [95\% CI: 1-1], while SpeeDX PCR displayed 90\% sensitivity (9/10) and 99\% specificity (105/106) Kappa $=0.89$ [95\% CI: $0.74-1]$. The pdhD PCR had 100\% sensitivity (10/10) and $100 \%$ specificity $(106 / 106)$ Kappa $=1$. The Aptima MG assay had $90 \%$ sensitivity (9/10) and 94\% specificity (100/106) Kappa $=0.69$ [0.46-0.91]. The SpeeDX 23S rRNA mutation rate was $50 \%(5 / 10)$. For the retrospective group, no Aptima MG was done because of transport media. A gold standard of 2 of 3 positive tests defined a true positive. The 16S PCR was $84 \%$ sensitive $(21 / 25)$ and $100 \%$ specific (264/264) Kappa $=0.91$ [0.813-0.997]. The SpeeDX was 92\% sensitive $(23 / 25)$ and $99 \%$ specificity $(262 / 264)$ Kappa $=0.91$ [0.8330.997]. The pdhD PCR was $96 \%$ sensitive (24/25) and $99 \%$ specific $(261 / 264)$ Kappa $=0.91$ [0.833-0.997]. The SpeeDX AZ resistance was 36\% (9/25).

Conclusion: More prospective evaluation studies are required; all assays performed well. The SpeeDX PCR compared well to two real-time PCRs and the Aptima MG assay. High AZ resistance was observed. The SpeeDX assay is potentially useful for MG diagnosis and for detection of resistance to $\mathrm{AZ}$

\section{P1.10 ORAL FLUID BASED RAPID SYPHILIS TESTING}

${ }^{1}$ Chelsea Shannon, ${ }^{2} \mathrm{CC}$ Bristow, ${ }^{1} \mathrm{SM}$ Herbst De Cortina, ${ }^{1} \mathrm{JJ}$ Chang, ${ }^{1} \mathrm{JD}$ Klausner. ${ }^{1}$ University of California, Los Angeles, Los Angeles, CA, USA; 'university of California, San Diego, La Jolla, CA, USA

10.1136/sextrans-2017-053264.118

Introduction Point-of-care syphilis testing currently uses fingerstick blood to identify Treponema pallidum (TP) antibodies by qualitative immunoassay. However, oral fluid testing is highly preferred by patients. We aimed to determine if rapid tests intended for whole blood could be used to detect treponemal antibody in oral fluid.

Methods Oral fluid was collected at the UCLA Care Clinic from 49 participants using Oasis SuperSAL (Vancouver, WA). The device uses an absorbent cylindrical pad to collect and filter $\sim 1 \mathrm{~mL}$ of oral fluid. Oral fluid filtrate was evaluated with 3 rapid syphilis tests: SD Bioline Syphilis 3.0 (SD), MedMira Reveal TP (MM), and First Response Anti-TP (FR) following manufacturer directions for whole blood. Reference test results, TP particle agglutination (TPPA) and rapid plasma reagin (RPR), were extracted from participants' medical records. We used 3 different definitions as a reference standard. Definition 1: TPPA reactive. Definition 2: TPPA and RPR reactive. Definition 3: TPPA reactive and RPR titer $>1: 4$. Specimens nonreactive on both the TPPA and RPR were considered negative. We calculated specificity and sensitivity for each definition, and used the exact binomial method to determine 95\% confidence intervals (CI).

Results With definitions 1,2 and 3 respectively, SD sensitivity was 86.4\% (CI: 65.1, 97.1), 93\% (CI: 66.1,99.8), 100\% (CI: 59.0,100); MM sensitivity was 6.3\% (CI: 0.2,30.2), 9.1\% (CI: $0.2,41.2$ ), $16.7 \%$ (CI: $0.4,64.1$ ); and FR sensitivity was $25 \%$ (CI: 8.7,49.1), 25\% (CI: 5.5,57.2), 14.3\% (CI: 0.4,57.9). SD specificity was $56 \%$ (CI: $36.5,75.5)$, MM specificity was 100\% (CI: 35.9,99.6) and FR specificity was 100\% (CI: $83.9,100)$.

Conclusion The high sensitivity of the SD test suggests a strong potential for oral fluid-based rapid syphilis testing. SD sensitivity increased with RPR titer. False positive results may be associated with the presence of non-venereal treponemal antibodies in oral fluid. The MM and FR tests had low sensitivity, perhaps due to differences in treponemal targets. Further research and development are needed.

\section{P1.11 DAMPENING OF THE MTRCDE EFFLUX PUMP OPERON IN NEISSERIA GONORRHOEAE STRAIN HO41 ENHANCES GONOCOCCAL SUSCEPTIBILITY TO PENICILLIN}

${ }^{1}$ Chen Sc, ${ }^{2} \mathrm{~S}$ Zugnhaier, ${ }^{3} \mathrm{M}$ Unemo, ${ }^{2}$ WM Shafer. ${ }^{1}$ National Centre for STD Control, Chinese Centre for Disease Control and Prevention, Nanjing - China Popular Republic; ${ }^{2}$ Departments of Microbiology and Immunology, Emory University School of Medicine, Atlanta, Georgia, USA; ${ }^{3}$ WHO Collaborating Centre for Gonorrhoea and Other STIS, Örebro - Sweden

\subsection{6/sextrans-2017-053264.119}

Introduction The resistance to extended-spectrum cephalosporins (ESCs), which is the only remaining antibiotic in many regions for treatment of gonorrhoea, expressed by certain strains of Neisseria gonorrhoeae is a major public concern worldwide. The first "superbug" reported in Japan was referred to H041, which was found to have high level resistance to ESCs and most other available antibiotics. Non-betalactamase resistance of gonococci to beta-lactam antibiotics requires several chromosomal mutations including a promoter mutation (single bp deletion in the mtrR promoter) that results in over-expression of the MtrCDE efflux. This promoter mutation results in loss of production of MtrR, which is the transcriptional repressor of $m t r C D E$., and is present in HO41. Here we investigated the possibility of reverting the resistant $\mathrm{HO} 41$ to be susceptible to $\beta$-lactam antibiotics by expressing MtrR and dampening the MtrCDE efflux pump. 